\title{
GMR
}

\section{Physiological evaluation of the responses of Larix olgensis families to drought stress and proteomic analysis of the superior family}

\author{
L. Zhang', H.G. Zhang ${ }^{1}$ and Q.Y. Pang ${ }^{2}$ \\ ${ }^{1}$ State Key Laboratory of Forest Genetics and Tree Breeding, \\ Northeast Forestry University, Harbin, China \\ ${ }^{2}$ Alkali Soil Natural Environmental Science Center, \\ Northeast Forestry University/Key Laboratory of Saline-Alkali Vegetation \\ Ecology Restoration in Oil Field, Ministry of Education, Harbin, China \\ Corresponding author: H.G. Zhang \\ E-mail: hanguozhang1@sina.com
}

Genet. Mol. Res. 14 (4): 15577-15586 (2015)

Received August 9, 2015

Accepted October 22, 2015

Published December 1, 2015

DOI http://dx.doi.org/10.4238/2015.December.1.9

ABSTRACT. The conifer Larix olgensis has been analyzed to delineate physiological and proteomic changes that occur under drought stress. Studies of the deleterious effects of drought in the larch families have mainly focused on photosynthesis. In the present study, when the intensity of drought was increased, plant height was inhibited as both POD and MDA levels increased, which indicates oxidative stress. Two-dimensional gel electrophoresis analysis detected 23 significantly differentially expressed proteins, of which 18 were analyzed by peptide mass fingerprinting by using MALDI-TOF/TOF. Eight spots were found to be up-regulated, while the other 10 spots were down-regulated during drought stress. The proteins that were induced by drought treatment have been implicated in the physiological changes that occurred. These results could provide additional information that could lead to a better understanding of the molecular basis of drought-sensitivity in larch plants.

Key words: Drought stress; Larix olgensis families; Proteomics 


\section{INTRODUCTION}

Water deficit is a major environmental stress that adversely affects plant physiological activity. Drought stress can cause biochemical and physiological responses in plants, such as growth inhibition, hyperosmotic stress, and oxidative damage. Larix olgensis A. Henry is one of the most important conifer species in China. It is a fast-growing timber species that makes up the largest afforestation area in northeast China. It is valued for its straight trunk, the tough, good quality of its lumber, and its durable qualities. We selected six excellent trees as both male and female parents to construct control-pollinated families, which allowed us to investigate the genetic possibilities that arose through the use of specific parents.

To survive drought stress, plants respond and adapt using complex developmental, morphological, physiological, and biochemical mechanisms. While the exact mechanisms have yet to be elucidated, peroxidases are known to increase plant defenses against pathogens (Karthikeyan et al., 2005). Peroxidases are sometimes used as histological markers. Cytochrome c peroxidase is used as a soluble, easily purified model for cytochrome c oxidase. MDA is a reactive aldehyde and one of the many reactive electrophile species that causes toxic stress to cells; MDA can form covalent protein adducts referred to as advanced lipoxidation end-products, which are analogous to advanced glycation end-products (Farmer and Davoine, 2007). The production of this aldehyde is used as a biomarker to measure the level of oxidative stress in an organism (Moore and Roberts, 1998; Del Rio et al., 2005). Proteomics have provided a new and useful way to identify the changes in protein expression that occur during drought stress. We can determine the molecular mechanism of the L. olgensis response to water deficit by analyzing physiological and proteomic alterations. Proteomic studies have already proven their value for assessing genetic variation (Bonhomme et al., 2009), the response to environment tolerance (Requejo and Tena, 2005; Pang et al., 2010), and bioinformatics (White et al., 2004) in various plant species.

In this study, we performed physiological evaluations and proteomic analysis of $L$. olgensis under drought stress.

\section{MATERIAL AND METHODS}

\section{Growth conditions and stress treatments}

L. olgensis trees from 22 control-pollinated families were produced by matings between six clones (Table 1). The parents LO73-1, LO73-2, LO73-3, LO73-14, LO73-22, and LO73-34 were obtained through asexual propagation of superior trees. Each family was planted in May 2008 and was grown under natural daylight and temperature conditions. Drought stress experiments were carried out on potted 4-year-old L. olgensis trees. Water was withheld until the desired soil water content was reached. The soil moisture for the pots of the well-watered and severe drought-stressed (25\%) groups were maintained with the required amounts of water by watering the plants daily and measuring the soil moisture with a soil moisture Meter (TDR300, Spectrum). After treatment for 15 and 30 days, the height of the stressed plants was measured. The needles were immediately frozen in liquid nitrogen and kept at $-80^{\circ} \mathrm{C}$ until further analysis. 
Table 1. Names and codes of Larix olgensis families.

\begin{tabular}{|c|c|c|c|c|c|}
\hline Family name & Family code & Family name & Family code & Family name & Family code \\
\hline LO73-1 x LO73-2 & $1 \times 2$ & LO73-1 x LO73-3 & $1 \times 3$ & LO73-1 x LO73-14 & $1 \times 14$ \\
\hline LO73-1 x LO73-34 & $1 \times 34$ & LO73-2 x LO73-1 & $2 \times 1$ & LO73-2 x LO73-2 & $2 \times 2$ \\
\hline LO73-2 x LO73-3 & $2 \times 3$ & LO73-2 x LO73-14 & $2 \times 14$ & LO73-2 x LO73-22 & $2 \times 22$ \\
\hline LO73-2 x LO73-34 & $2 \times 34$ & LO73-3 x LO73-1 & $3 \times 1$ & LO73-3 x LO73-2 & $3 \times 2$ \\
\hline LO73-3 x LO73-3 & $3 \times 3$ & LO73-3 x LO73-14 & $3 \times 14$ & LO73-14 x LO73-3 & $14 \times 3$ \\
\hline LO73-14 x LO73-14 & $14 \times 14$ & LO73-14 x LO73-34 & $14 \times 34$ & LO73-22 x LO73-14 & $22 \times 14$ \\
\hline LO73-34 x LO73-1 & $34 \times 1$ & LO73-34 x LO73-2 & $34 \times 2$ & LO73-34 x LO73-14 & $34 \times 14$ \\
\hline LO73-34 x LO73-34 & $34 \times 34$ & & & & \\
\hline
\end{tabular}

\section{Determination of antioxidant enzyme activity}

Guaiacol peroxidase (POD, EC1.11.1.7) activity was assayed by determining the level of guaiacol oxidation by $\mathrm{H}_{2} \mathrm{O}_{2}$ through changes in absorbance changes $470 \mathrm{~nm}$, as described by Castillo et al. (1984), with some modifications. The $3-\mathrm{mL}$ reaction mixture contained 100 $\mathrm{mM}$ potassium phosphate buffer, $\mathrm{pH}$ 7.0, $20 \mathrm{mM}$ guaiacol, $10 \mathrm{mM} \mathrm{H}_{2} \mathrm{O}_{2}$, and $50 \mathrm{~mL}$ enzyme (approximately $30 \mathrm{mg}$ protein). The changes in the absorbance of the reaction solution at 470 nm were determined for 1 min.

\section{Analysis of lipid peroxidation}

MDA is a final decomposition product of lipid peroxidation and has been used as an index of lipid peroxidation status. The MDA content was determined using the thiobarbituric acid (TBA) reaction, as described by Stewart and Bewley (1980), with some modifications. One gram of needles (FW) was homogenized in $4 \mathrm{~mL} 0.5 \%(\mathrm{w} / \mathrm{v})$ TCA. The homogenate was centrifuged at $10,000 \mathrm{~g}$ for $10 \mathrm{~min}$, and $4 \mathrm{~mL} 20 \%$ TCA containing $0.5 \%$ (w/v) TBA was added to $1 \mathrm{~mL}$ supernatant. The mixture was heated at $95^{\circ} \mathrm{C}$ for $20 \mathrm{~min}$ and quickly cooled on ice; the mixture was then centrifuged at $10,000 \mathrm{~g}$ for $10 \mathrm{~min}$, and the absorbance of the supernatant was read at 532 and $600 \mathrm{~nm}$. After subtracting the non-specific absorbance recorded at 600 $\mathrm{nm}$, the MDA concentration was determined using its extinction coefficient of $155 \mathrm{mM} / \mathrm{cm}$.

\section{Protein extraction and 2-DE analysis}

Protein extraction was performed according to the method described by Damerval et al. (1986) and Kamo et al. (1995), with some modifications. The needles were ground in liquid nitrogen and incubated in $10 \%(\mathrm{w} / \mathrm{v})$ TCA/acetone containing $0.07 \%(\mathrm{w} / \mathrm{v})$ DTT at $-20^{\circ} \mathrm{C}$ for $1 \mathrm{~h}$; the mixtures were then centrifuged at $35,000 \mathrm{~g}$ for $15 \mathrm{~min}$. The pellets were washed with ice-cold acetone containing $0.07 \%$ DTT, incubated at $-20^{\circ} \mathrm{C}$ for $1 \mathrm{~h}$, and centrifuged at 12,000 $g$ for $15 \mathrm{~min}$. Two washing steps were performed, and the pellets were dried in a vacuum centrifuge. The resultant powder was resuspended in a solubilization buffer consisting of $9 \mathrm{M}$ urea, $4 \%(\mathrm{w} / \mathrm{v})$ CHAPS, $1 \%(\mathrm{w} / \mathrm{v})$ DTT, $1 \%(\mathrm{v} / \mathrm{v})$ ampholytes, $\mathrm{pH} 4-7$, and $35 \mathrm{mM}$ Tris base. The total protein concentration was quantified using a 2-D gel protein quantification kit (GE Healthcare, USA).

Isoelectric focusing was performed using the IPGphor system (GE Healthcare). Each sample, which contained $1 \mathrm{mg}$ total protein, was loaded onto 24-cm IPG gel strips, $\mathrm{pH} 4-7$, and run at $30 \mathrm{~V}$ for $8 \mathrm{~h}$, at $50 \mathrm{~V}$ for $4 \mathrm{~h}$, then at $100,300,500$, and $1000 \mathrm{~V}$ for $1 \mathrm{~h}$ each, followed by 
$8000 \mathrm{~V}$ for $12 \mathrm{~h}$. The second dimension was performed on 12.5\% SDS-PAGE using an Ettan DALTsix Electrophoresis Unit (GE Healthcare) at $2 \mathrm{~W} / \mathrm{gel}$ for $30 \mathrm{~min}$, then at $15 \mathrm{~W} / \mathrm{gel}$ for about $5 \mathrm{~h}$ at $16^{\circ} \mathrm{C}$. The gels were stained with Coomassie Brilliant Blue R250 according to a protocol described by Shevchenko et al. (1996). At least three replicates were performed for each sample. The analytical gel images were acquired using an ImageScanner (GE Healthcare), and image analysis was performed using the PDQuest 2-DE analysis software (Bio-Rad).

\section{In-gel digestion and MALDI-TOF mass spectrometry (MS) analysis}

Protein spots were excised from the preparative gels, washed three times with ultrapure water, destained twice with $50 \mathrm{mM} \mathrm{NH}_{4} \mathrm{HCO}_{3}$ in $50 \%$ acetonitrile, reduced with 10 $\mathrm{mM}$ DTT in $50 \mathrm{mM} \mathrm{NH}_{4} \mathrm{HCO}_{3}$, alkylated with $40 \mathrm{mM}$ iodoacetamide in $50 \mathrm{mM} \mathrm{NH}_{4} \mathrm{HCO}_{3}$, dried twice with $100 \%$ acetonitrile, and digested overnight at $37^{\circ} \mathrm{C}$ with sequencing grademodified trypsin (Promega, Madison, WI, USA) in $50 \mathrm{mM} \mathrm{NH}_{4} \mathrm{HCO}_{3}$. The peptides were extracted twice with $0.1 \%$ TFA in $50 \%$ acetonitrile. The extracts were pooled and lyophilized. The resulting lyophilized tryptic peptides were dissolved in $5 \mathrm{mg} / \mathrm{mL} \mathrm{CHCA}$ containing $0.1 \%$ TFA and $50 \%$ acetonitrile. MS analysis was conducted using a MALDI-TOF/TOF MS 4700 Proteomics Analyzer (Applied Biosystems, Framingham, MA, USA). The data were analyzed using the GPS Explorer software (Applied Biosystems) and the MASCOT software (Matrix Science, London, UK). NCBInr and green plant were selected as the database and taxonomy, respectively.

\section{RESULTS}

\section{Plant growth response}

Drought stress inhibited the height growth of all families, and the experimental plants grew less than the control plants did. During the extension of drought stress, all families exhibited slower growth than was observed at the beginning of stress treatment (Figure 1). The average height of all families decreased by $1.91 \%$ after 15 days and by $8.66 \%$ after 30 days. The $2 \times 2,34 \times 2$, and $14 \times 3$ families were the three families with the most growth when grown under drought stress conditions for 30 days. The height growth of these three families decreased by $4.05,4.28$, and $4.32 \%$ compared to the control, respectively.

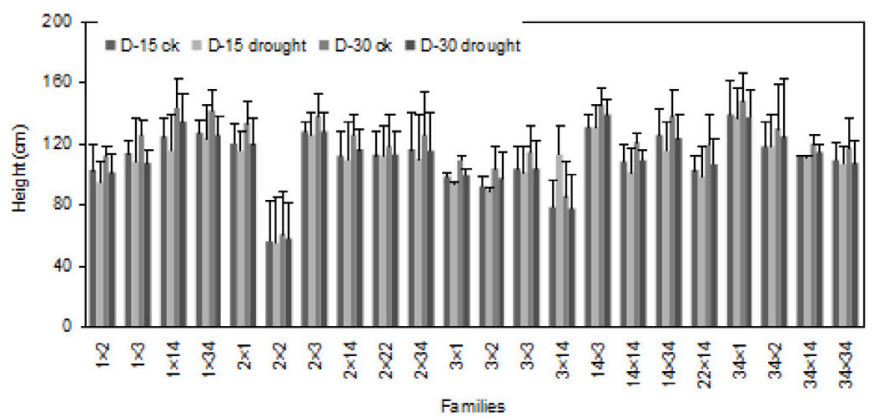

Figure 1. Height growth of different Larix olgensis families under well-watered and drought stress conditions on days 15 and 30 . 
The MDA and peroxide content reflects the extent of cellular damage; MDA levels increased and POD activity decreased during the period of drought extension.

Drought caused a significant decrease in POD activity after 15 days $(\mathrm{P}<0.05)$ and a highly significant decrease after 30 days $(\mathrm{P}<0.01)$. The POD activity of some families increased slightly after 15 days, while all activities decreased after 30 days (Figure 2). The 14 $\times 3,34 \times 2$, and $3 \times 1$ families were the three families whose POD activity decreased the least during the 30 days. The POD activities of these families decreased by $0.48,5.20$, and $11.0 \%$ compared to those of the control, respectively. MDA concentration significantly increased after 30 days $(\mathrm{P}<0.01$; Figure 3$)$. Some families exhibited minimal variation between the control and drought-treated samples, and these trends were not the same as the average changes in POD activity. The $34 \times 14,34 \times 2$, and $1 \times 14$ families were the three families whose MDA content increased the least during the 30 days; the MDA content of these families increased by $0.06,0.75$, and $1.70 \%$ compared to that of the control, respectively. The average increase in MDA content in these three families was significantly lower than the total average.

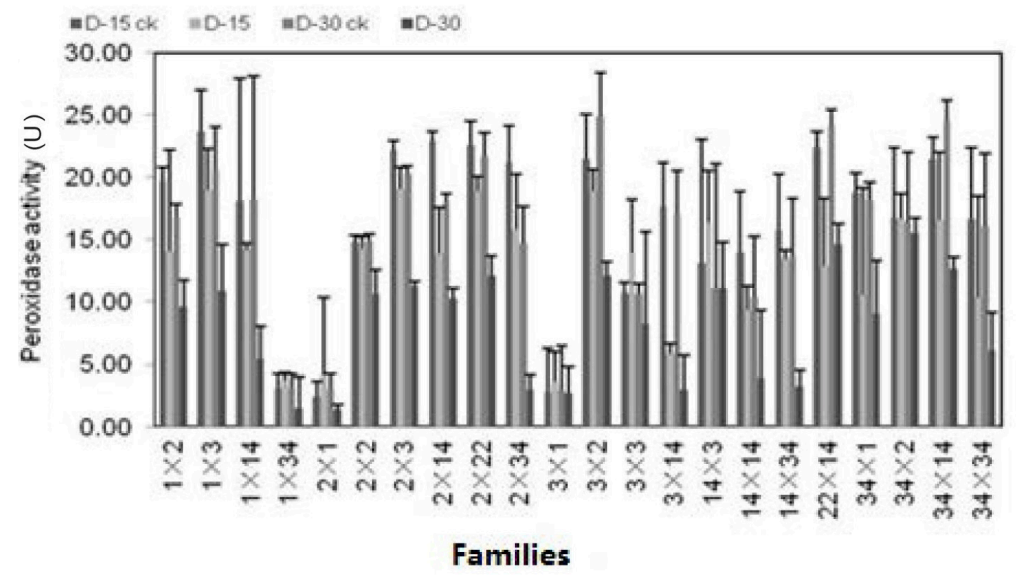

Figure 2. Peroxidase activity of the different Larix olgensis families under well-watered and drought stress conditions on days 15 and 30 .

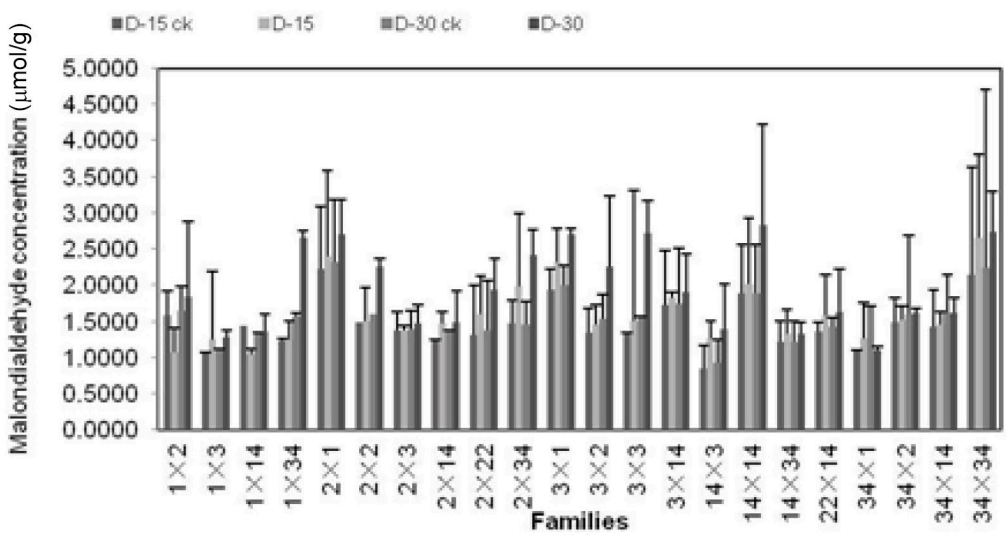

Figure 3. MDA content of the different families under well-watered and drought stress conditions on days 15 and 30. 


\section{Drought-responsive proteins in the superior family}

To investigate changes in the protein expression profiles of $L$. olgensis under drought stress, we performed 2-DE analysis of the total protein content of three biological replicates of conifers from the $34 \times 2$ family; at least three gels were assessed for each sample, and high reproducibility was observed. According to the morphological changes, and growth and physiological responses to drought conditions, we selected the $34 \times 2$ family from which to obtain material for further investigation of differentially expressed proteins. A representative gel utilizing material from L. olgensis is shown in Figure 4, and the positions of the differentially expressed spots are marked.

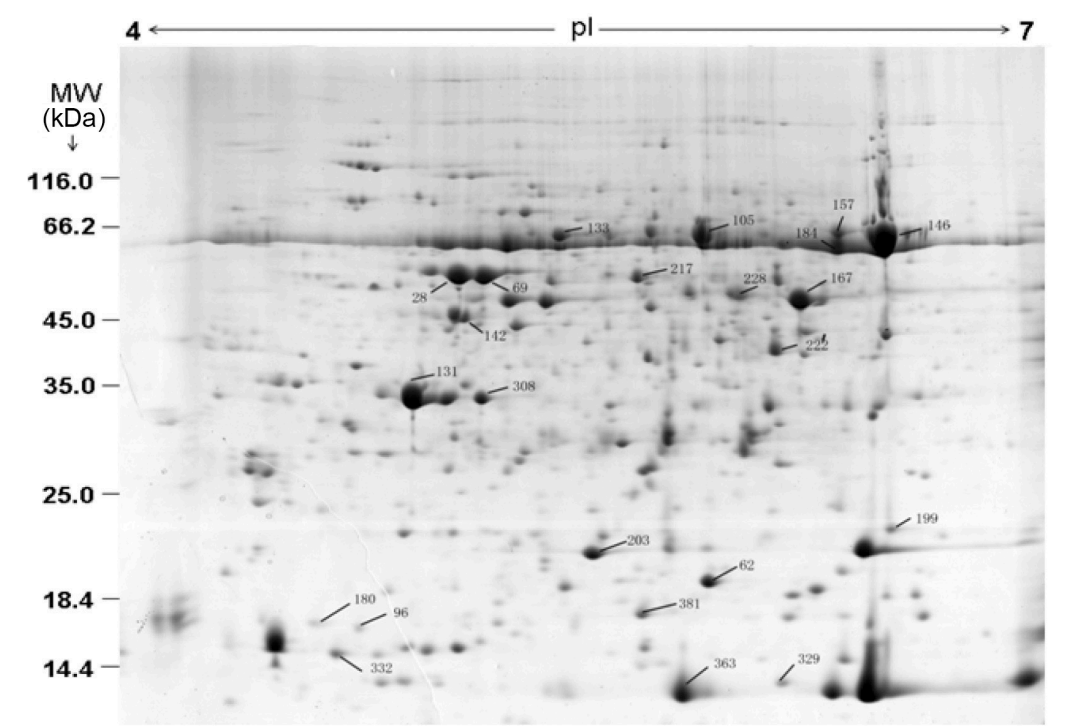

Figure 4. Soluble conifer proteins subjected to 2-D-PAGE analysis (first dimension: IEF, pH 4-7; second dimension: SDS-PAGE $12.5 \%$ ), and colloidal Coomassie blue staining.

Overall, drought stress induced significant variation in the abundance of 23 spots, all of which were submitted to MS/MS (MALDI-TOF-TOF, ABI-4800) analysis. Among the proteins tested, 18 protein spots $(78 \%)$ were reliably identified; their functions are listed in Table 2. However, the peptide maps for most of the remaining spots only matched to proteins of other species. It is clear that the sequences of the L. olgensis proteins present in these spots were not present in the protein database. Among all of the spots (Figure 5), we found that some proteins were up-regulated, including ATP synthase CF1 alpha subunit (spot No. 105), cytosolic glutamine synthetase (spot No. 167), Cu/Zn superoxide dismutase II (spot No. 62), chloroplast elongation factor TuA (spot No. 217), oxygen evolving enhancer protein 1 (spot No. 308), plastidic aldolase (spot No. 222), myosin-like protein (spot No. 142), and one unknown protein (spot No. 131). Other proteins were down-regulated, including ribulose-1,5bisphosphate carboxylase/oxygenase (RuBisCO) large subunit (spot Nos. 146, 199, 203, 184, 381), RuBisCO activase (spot Nos. 28, 69), putative ATP synthase beta subunit (spot No. 133), RuBisCO large subunit (spot No. 157), and a putative retro-element (spot No. 96). 
Physiological evaluation of Larix to drought

15583

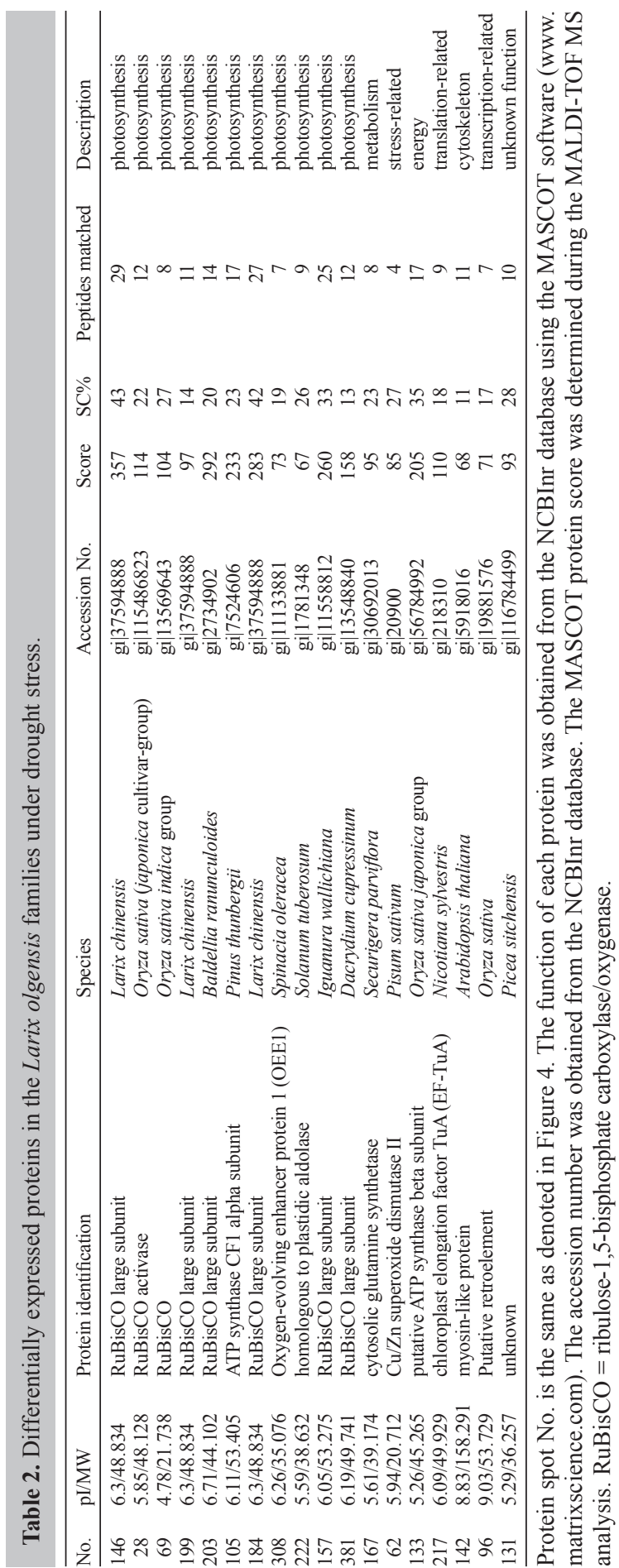

Genetics and Molecular Research 14 (4): 15577-15586 (2015)

CFUNPEC-RP www.funpecrp.com.br 


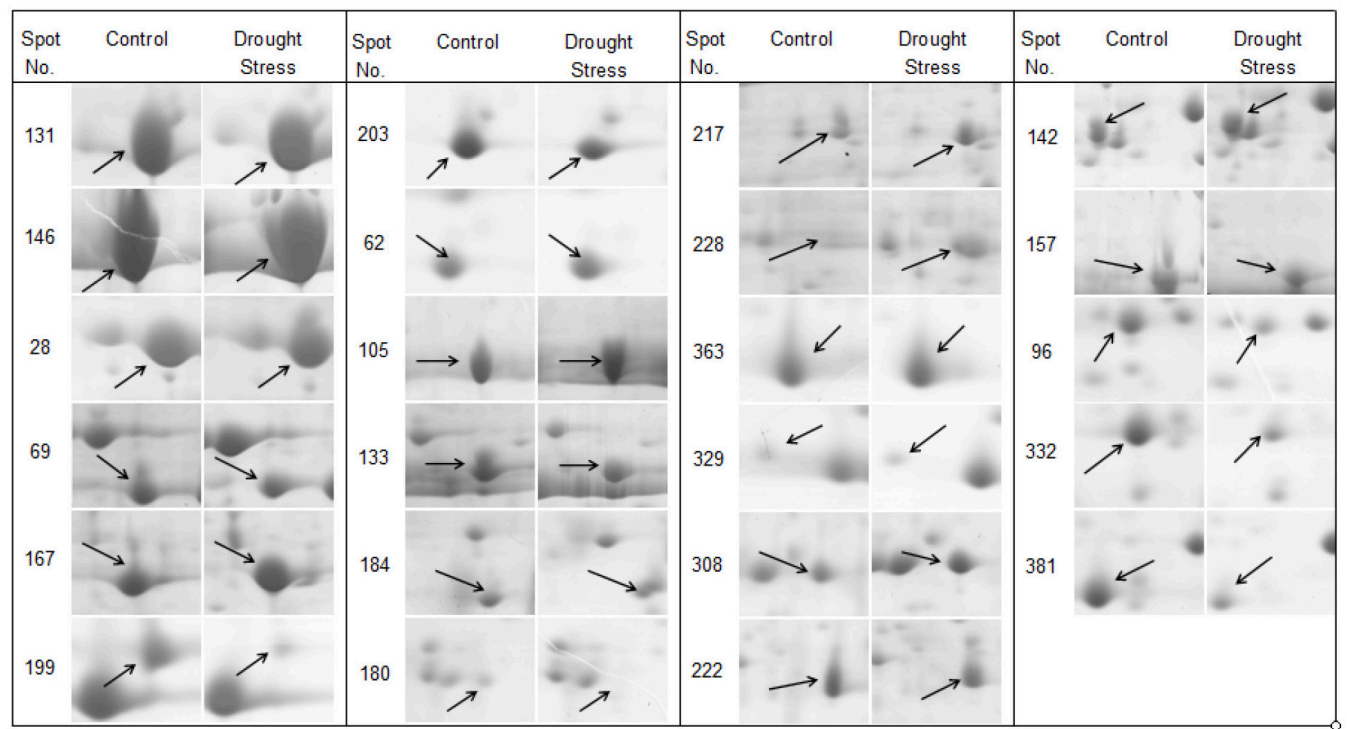

Figure 5. Changes in protein abundance in the leaves of two contrasting Larix olgensis samples from well-watered (control) and water-stressed specimens.

To further examine the differentially expressed proteins, the identified proteins were grouped by their functions (Table 2). The largest functional category contained proteins involved in photosynthesis $(61 \%)$, and the others consisted of metabolism-, stress-, energy-, translation-, cytoskeleton-, and transcription-related proteins and those of unknown function. Thus, the main proteins expressed under drought stress were those related to photosynthesis.

\section{DISCUSSION}

Responses of plants to drought stress depend on the species, genotype, drought duration, severity of the water deficit, and the age and developmental stage of the plant. MDA is an end-product of lipid peroxidation, and MDA levels reflect the degree of peroxidation of the membrane lipids. $\mathrm{H}_{2} \mathrm{O}_{2}$, as an ROS, can damage membrane lipids, proteins, and DNA (Foyer and Noctor, 2009).

In this study, we investigated the responses of $L$. olgensis families to severe drought stress (25\% soil water content). Trends in the growth and physiological responses to drought stress were similar among the different families. Drought stress significantly inhibited growth and increased MDA content, while it decreased POD activity after 30 days. Physiological changes at the cellular level that are associated with drought stress typically include a reduction in plant growth, inhibition of photosynthetic activity, increased osmosis, and changes in carbohydrate metabolism (Xiao et al., 2009). Similar results have been reported in previous studies (Wang et al., 2007; Gao et al., 2009).

The 2-D-PAGE approach remains one of the most common tools used to investigate the differential expression of proteins. Although this approach has its limitations, including hydrophobic membranes, proteins with extreme pI, and difficulty in detecting proteins present 
at low abundance in complex protein mixtures, it is widely used for proteome analysis (Fulda et al., 2011).

The different families experienced the same level of water deficit, and similar growth and physiological responses were observed under water deficit conditions that were used to simulated drought stress. Therefore, we selected the $34 \times 2$ family, which exhibited a slight increase in MDA content, a slight decline in POD activity, and decreased growth inhibition under drought stress. Proteomic analyses showed that there were significant differences in protein expression patterns. The present proteomic study identified some drought stressresponsive proteins, including those induced by stress, and those related to photosynthesis, energy, translation, and transcription. Eleven proteins associated with photosynthesis were found to be differentially expressed in L. olgensis conifers under drought stress.

$\mathrm{RuBisCO}$ is involved in the first major step of carbon fixation, a process by which plants convert atmospheric carbon dioxide into energy-rich molecules, such as glucose (Feller et al., 2008). $\mathrm{RuBisCO}$ is regulated by $\mathrm{RuBisCO}$ activase, which is required for the rapid formation of the critical carbamate in the active site of RuBisCO in plants and some algae (Portis Jr., 2003).

ATP synthase CF1 is usually tightly coupled to the thylakoid membrane and catalyzes the conversion of ADP to ATP using the electrochemical potential generated during photosynthetic electron transport. The glutamine-synthetase isoform is found in the cytosol and does not appear to be induced by light (Sakamoto et al., 1990). Cu,Zn-superoxide dismutase II (Cu,Zn-SOD2) was found to be functional in an in vitro eukaryotic system and could modulate the redox status of tumor cells (Hadji Sfaxi et al., 2012). Myosins are a large family of actin-based motor proteins that are involved in a variety of cellular processes (Landsverk and Epstein, 2005).

The down-regulation and inhibition of photosynthesis during water stress could be explained, at least in part, by a decrease in the levels of RuBisCO subunits and the activase protein. Similar results have also been reported in cotton (Deeba et al., 2012) and poplar (Xiao et al., 2009). A similar down-regulation of the ATP synthase chain was reported in sugarcane leaves under osmotic stress (Zhou et al., 2012). These data show that photosynthesis was inhibited under drought stress in an excellent larch family, and that osmoregulation was affected at the cytoplasmic level.

\section{Conflicts of interest}

The authors declare no conflict of interest.

\section{ACKNOWLEDGMENTS}

Research supported by the Fundamental Research Funds for the Central Universities (\#DL13BAX12) and the National Science and Technology Support Program "The Breeding Research of Larch and Spruce in Cold Temperate" (\#2012BAD01B0102).

\section{REFERENCES}

\footnotetext{
Bonhomme L, Monclus R, Vincent D, Carpin S, et al. (2009). Genetic variation and drought response in two Populus x euramericana genotypes through 2-DE proteomic analysis of leaves from field and glasshouse cultivated plants. Phytochemistry 70: 988-1002.

Castillo FJI, Penel CI and Greppin H (1984). Peroxidase release induced by ozone in Sedum album leaves: Involvement
} 
of Ca. Plant Physiol. 74: 846-851.

Damerval C, De Vienne D, Zivy M and Thiellement H (1986) The technical improvements in two-dimensional electrophoresis increase the level of genetic variation detected in wheat-seedling proteins. Electrophoresis 7: 52-54.

Deeba F, Ashutosh K, Pandey AK, Ranjan S, et al. (2012). Physiological and proteomic responses of cotton (Gossypium herbaceum L.) to drought stress. Plant Physiol. Biochem. 53: 6-18.

Del Rio D, Stewart AJ and Pellegrini N (2005). A review of recent studies on malondialdehyde as toxic molecule and biological marker of oxidative stress. Nutr. Metab. Cardiovasc. Dis. 15: 316-328.

Farmer EE and Davoine C (2007). Reactive electrophile species. Curr. Opin. Plant Biol. 10: 380-386.

Feller U, Anders I and Mae T (2008). Rubiscolytics: fate of Rubisco after its enzymatic function in a cell is terminated. $J$. Exp. Bot. 59: 1615-24.

Foyer CH and Noctor G (2009). Redox regulation in photosynthetic organisms: signaling, acclimation, and practical implications. Antioxid. Redox Signal. 11: 861-905.

Fulda S, Mikkat S, Stegmann H and Horn R (2011). Physiology and proteomic of drought stress acclimation in sunflower (Helianthus annuus L.). Plant Biol. 13: 632-642.

Gao D, Gao Q, Xu HY, Ma F, et al. (2009). Physiological responses to gradual drought stress in the diploid hybrid Pinus densata and its two parental species. Trees 23: 717-728.

Hadji Sfaxi I, Ezzine A, Coquet L, Cosette P, et al. (2012). Combined Proteomic and molecular approaches for cloning and characterization of copper-zinc superoxide dismutase (Cu, Zn-SOD2) from Garlic (Allium sativum). Mol. Biotechnol. 52: 49-58.

Kamo M, Kawakami T, Miyatake N and Tsugita A (1995). Separation and characterization of Arabidopsis thaliana proteins by two-dimensional gel electrophoresis. Electrophoresis 16: 423-430.

Karthikeyan M, Jayakumar V, Radhika K, Bhaskaran R, et al. (2005). Induction of resistance in host against the infection of leaf blight pathogen (Alternaria palandui) in onion (Allium cepa var aggregatum). Indian J. Biochem. Biophys. 42: 371-377.

Landsverk ML and Epstein HF (2005). Genetic analysis of myosin II assembly and organization in model organisms. Cell Mol. Life Sci. 62: 2270-2282.

Moore K and Roberts LJ 2nd (1998). Measurement of lipid peroxidation. Free Radic. Res. 28: 659-671.

Pang Q, Chen S, Dai S, Chen Y, et al. (2010). Comparative proteomics of salt tolerance in Arabidopsis thaliana and Thellungiella halopila. J. Proteome Res. 9: 2584-2599.

Portis AR Jr (2003). Rubisco activase-Rubisco's catalytic chaperone. Photosynth. Res. 75: 11-27.

Requejo R and Tena M (2005). Proteome analysis of maize roots reveals that oxidative stress is a main contributing factor to plant arsenic toxicity. Phytochemistry 66: 1519-1528.

Sakamoto A, Takeba G, Shibata D and Tanaka K (1990). Phytochrome-mediated activation of the gene for cytosolic glutamine-synthetase (GS, ) during imbibition of photosensitive lettuce seeds. Plant Mol. Biol. 15: 317-323.

Shevchenko A, Wilm M, Vorm O and Mann M (1996). Mass spectrometric sequencing of proteins silver-stained polyacrylamide gels. Anal. Chem. 68: 850-858.

Stewart RRC and Bewley JD (1980). Lipid peroxidation associated with accelerated aging of soybean axes. Plant Physiol. 65: 245-248.

Xiao X, Yang F, Zhang S, Korpelainen H, et al. (2009). Physiological and proteomic responses of two contrasting Populus cathayana populations to drought stress. Physiol. Plant 136: 150-168.

Wang R, Chen S, Deng L, Fritz E, et al. (2007). Leaf photosynthesis, fluorescence response to salinity and the relevance to chloroplast salt compartmentation and anti-oxidative stress in two poplars. Trees 21: 581-591.

White NC, Chan DW and Zhang Z (2004). Bioinformatics strategies for proteomic profiling. Clin. Biochem. 37: 636-641.

Zhou G, Yang LT, Li YR, Zou CL, et al. (2012). Proteomic analysis of osmotic stress-responsive proteins in sugarcane leaves. Plant Mol. Biol. Rep. 30: 249-259. 